\title{
STRUCTURES ARGYROPHILES SUPERFICIELLES DU MIRACIDIUM ET DE LA CERCAIRE DE PARAMPHISTOMUM LEYDENI NASMARK, 1937.
}

\author{
P. SAMNALIEV*, L. A. PINO**, Ch. BAYSSADE-DUFOUR** et J.-L. ALBARET**
}

RÉSUMÉ. Le cycle biologique de Paramphistomum leydeni Näsmark, 1937 est reproduit en laboratoire. Les adultes proviennent de la panse d'un Bos taurus naturellement infesté de Tchécoslovaquie. Des Planorbis planorbis sains infestés expérimentalement émettent les premières cercaires deux mois après l'infestation.

Les structures argyrophiles du miracidium et de la cercaire sont décrites. Celles du miracidium sont identiques à celles de tous les miracidiums des espèces du genre Paramphistomum actuellement décrites dont $P$. togolense, $P$. phillerouxi, $P$. daubneyi et $P$. microbothrium; elles sont différentes de celles du miracidium de Calicophoron calicophorum. Les structures argyrophiles de la cercaire ont des caractéristiques communes avec celles des autres cercaires de Paramphistomum déjà décrites : $P$. togolense, $P$. phillerouxi, $P$. daubneyi et $P$. microbothrium en particulier aux niveaux $\mathrm{C}_{\mathrm{II}}$ et $\mathrm{AM} \mathrm{V}$, mais la chétotaxie acétabulaire très particulière permet de différencier $P$. leydeni des autres cercaires de Paramphistomum connues à ce jour.

Ainsi, d'une part, chaque espèce est aisément différenciable des espèces voisines, d'autre part, le genre Paramphistomum tel qu'il est admis par Näsmark (1937) apparaît bien fondé.

\section{Superficial argentophilic structures of miracidium and cercaria of Param- phistomum leydeni Näsmark, 1937.}

SUMMARY. The life cycle of Paramphistomum leydeni Näsmark, 1937, is carried out in the laboratory. Adults were obtained from the rumen of a naturally infected Bos taurus in Czechoslovakia. Laboratory-reared Planorbis planorbis experimentally infected shed the first cercariae two months after exposure to miracidia.

Argentophilic structures in miracidia and cercariae are described. In miracidia, these structures are similar to those previously described from species of Paramphistomum : P. togolense, P. phillerouxi, P. daubneyi, P. microbothrium. They differ, however, from those of Calicophoron calicophorum.

In cercariae, argentophilic structures have some features in common with those of the species of Paramphistomum mentioned above, especially at the levels $\mathrm{C}_{\mathrm{II}}$ and AM V, but the very peculiar acetabular chaetotaxy allows us to distinguish $P$. leydeni from these species.

Thus, each species can easily be differentiated from the related species; moreover the genus Paramphistomum as characterized by Näsmark (1937) appears to be justified.

* Bulgarian Academy of Sciences, Central Laboratory of Helminthology SOFIA 1113, Kv « Geo Milev », Akad. G. Bontchev str. bl. 25 Bulgaria.

** Laboratoire des Vers associé au CNRS, Muséum National d'Histoire Naturelle, 61 rue de Buffon, F 75231 Paris Cedex 05 et Laboratoire d'Helminthologie de l'École des Hautes Études.

Accepté le 8 juin 1983 . 


\section{Estructuras argirófilas superficiales del miracidio y de la cercaria de Paramphistomum leydeni Näsmark, 1937}

RESUMEN Se realiza el ciclo biológico de Paramphistomum leydeni Näsmark, 1937 en condiciones de laboratorio. Los adultos se obtuvieron de un Bos taurus naturalmente infestado proveniente de Checoslovaquia. Se infestaron experimentalmente ejemplares de Planorbis planorbis sanos y se obtienen dos meses despues las cercarias correspondientes.

Se describen las estructuras argirófilas del miracidio y de la cercaria. Las del miracidio son identicas a aquellas de todos los de las especies del género Paramphistomum conocidas : P. tologense, $P$. phillerouxi, $P$. daubneyi y $P$. microbothrium; dichas estructuras son diferentes de las del miracidio de Calicophoron calicophorum. Las estructuras argirófilas de la cercaria tienen caracteristicas comunes a las de otras cercarias de Paramphistomum ya descritas: P. togolense, P. phillerouxi, $P$. daubneyi y $P$. microbothrium en particular en los niveles $\mathrm{C}_{\mathrm{II}}$ y AM V, pero la quetotaxia acetabular muy particular, permite diferenciar a $P$. leydeni de las otras cercarias de Paramphistomum conocidas hasta el presente.

Asi, se concluye que cada especie es facilmente diferenciable de las especies vecinas y que el género Paramphistomum tal como lo admite Näsmark (1937), parece bien fundamentado.

Nous décrivons les structures argyrophiles des miracidiums et cercaires d'une souche de Paramphistomes obtenue à partir de Vers adultes infestant à l'état naturel un Bos taurus de Tchécoslovaquie.

Les miracidiums sont en partie utilisés pour l'imprégnation argentique et en partie utilisés par l'un de nous (Samnaliev) pour l'infestation expérimentale de Planorbis planorbis sains, ces Mollusques émettent deux mois après l'infestation les cercaires que nous étudions ci-dessous.

Les adultes sont déterminés par les Docteurs Vétérinaires tchécoslovaques Pacenovsky et Krupicer : Liorchis scotiae. Or, tout récemment, Eduardo (1982a, b) se basant à la fois sur les données d'histologie classique et sur un nouvel élément de diagnose : l'étude du tégument des Paramphistomes adultes au microscope à balayage, montre, d'une part que le genre Liorchis n'est pas valide mais synonyme de Paramphistomum, d'autre part, que l'espèce scotiae est synonyme de leydeni. Nous ne disposons d'aucun argument pour mettre en doute ses conclusions et désignons donc notre matériel sous le nom de Paramphistomum leydeni Näsmark, 1937.

\section{I - Structures argyrophiles du miracidium (fig. 1)}

Les miracidiums sont imprégnés au nitrate d'argent selon la méthode de Lynch (1933), ils montrent : des cellules épidermiques ciliées en nombre et disposition classiques c'est-à-dire au nombre de 20 réparties d'avant en arrière en quatre étages de 6, 8, 4 et 2 cellules séparées par trois ceintures : antérieure, moyenne et postérieure. 

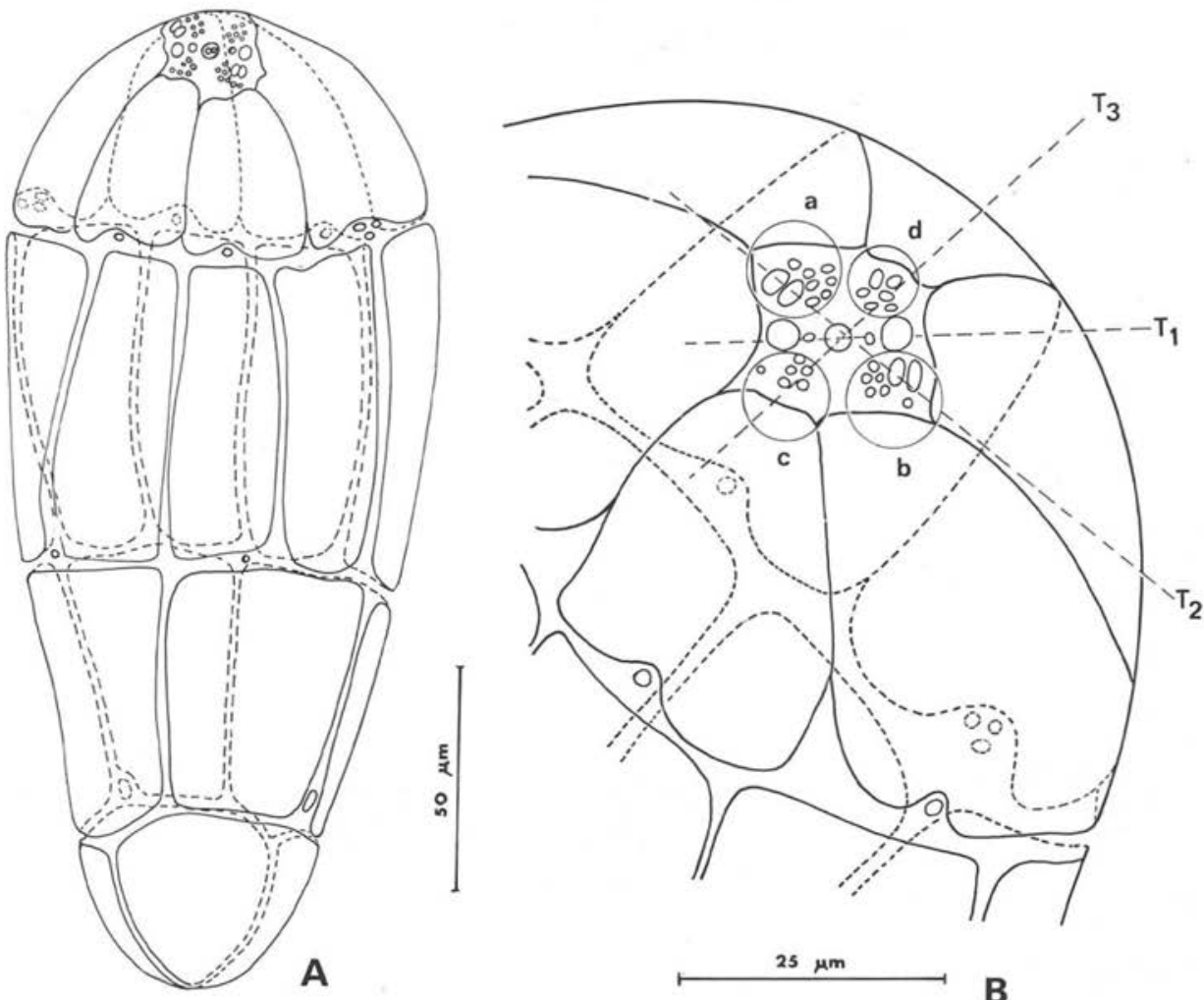

FIG. I. - Structures argyrophiles superficielles du miracidium de Paramphistomum leydeni. A) Vue d'ensemble ; B) Détail du térébratorium.

Les organites argyrophiles superficiels s'observent à quatre niveaux : le térébratorium, la ceinture antérieure, la ceinture moyenne et la ceinture postérieure.

- Le térébratorium porte une trentaine de formations réparties selon trois alignements : T1, T2 et T3. Selon T1, confondus avec le plan frontal, se situent, de part et d'autres d'une formation centrale, deux organite dont un plus petit. Selon T2, les groupes " $\mathrm{a}$ » et " $\mathrm{b}$ » sont constitués chacun de 8 à 9 éléments dont 2 plus grands contigus. Selon T3, les groupes " $\mathrm{c}$ » et « $\mathrm{d}$ » comprennent chacun 6 à 7 formations.

- La ceinture antérieure porte un organite dans une échancrure à la base de chaque cellule ciliée du premier étage et latéralement deux éléments supplémentaires, soit un total de 10 organites argyrophiles.

- La ceinture moyenne porte deux formations symétriques par rapport au plan sagittal; elles sont séparées par un angle de $90^{\circ}$.

- Sur la ceinture postérieure s'ouvrent latéralement deux pores excréteurs. 


\section{II - Structures argyrophiles de la cercaire (fig. 2 et 3 )}

Les cercaires sont imprégnées au nitrate d'argent selon la méthode de Combes et coll. (1976); les structures argyrophiles sont réparties en quatre régions :

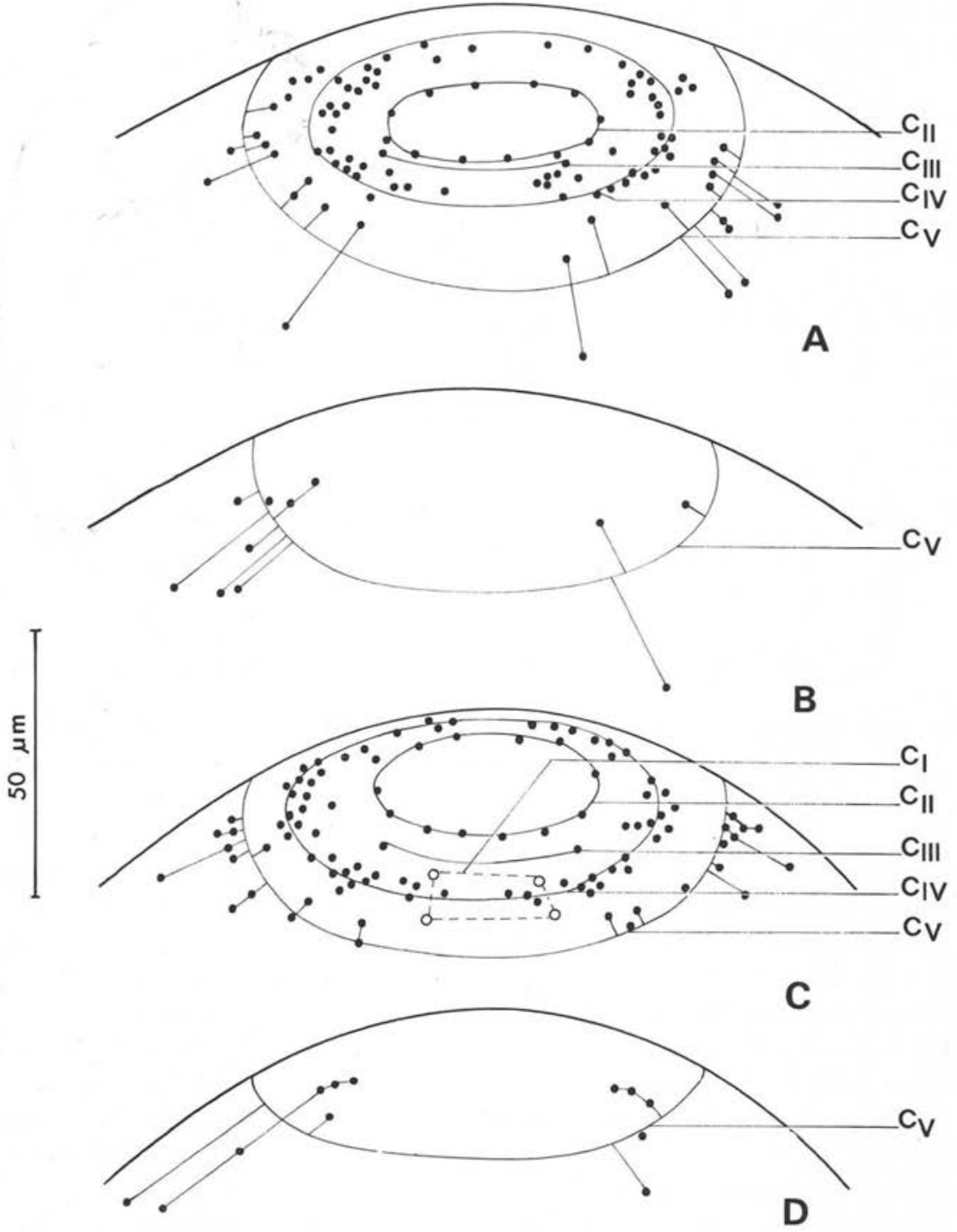

FIG. 2. - Chétotaxie céphalique de la cercaire de Paramphistomum leydeni. A) C) : vues ventrales; le cycle $C_{1}$ n'est pas représenté en $A$; ses papilles sont représentées en cercles clairs en $\mathrm{C}$; B) D) : vues dorsales. 
- Région céphalique (fig. 2)

Elle porte au total 118 à 126 papilles réparties selon cinq cycles :

$\mathrm{C}_{\mathrm{I}}=4$ papilles invaginées

$\mathrm{C}_{\mathrm{II}}=12$ papilles

$\mathrm{C}_{\mathrm{III}}=2$ papilles

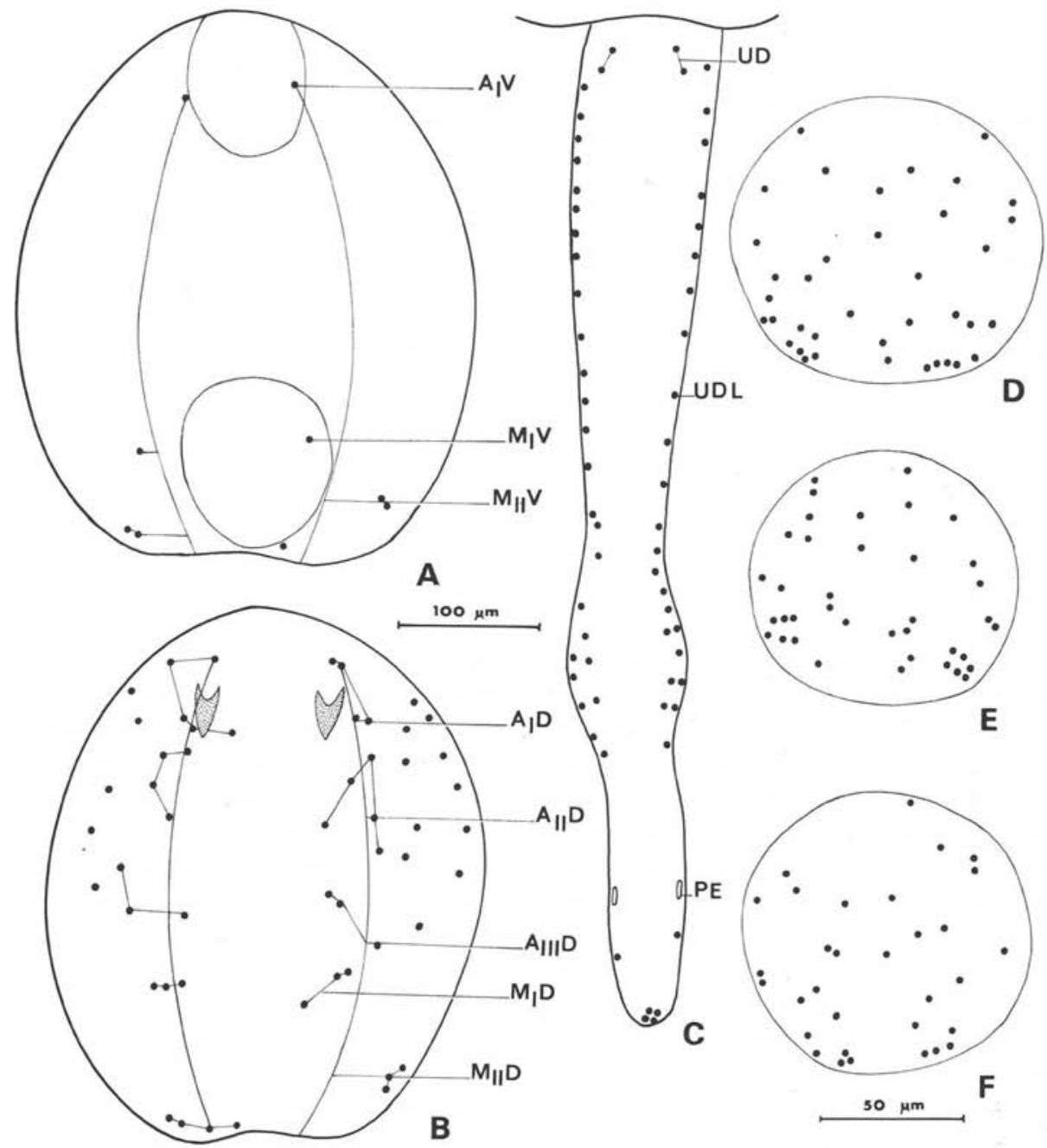

FIG. 3. - Chétotaxies corporelle caudale et acétabulaire de la cercaire de Paramphistomum leydeni.

A) Papilles corporelles ventrales; B) Papilles corporelles dorsales et latérales; C) Papilles caudales dorsales, dorsolatérales et terminales (en clair P. E. = pores excréteurs) ; D) E) F) Papilles acétabulaires. 
$\mathrm{C}_{\mathrm{IV}}=47$ à 52 papilles

$\mathrm{C}_{\mathrm{V}}=43$ à 51 papilles

- Région corporelle (fig. $3 A, B$ )

Elle porte au total 56 à 72 papilles, soit par hémicorps :
$\mathrm{A}_{\mathrm{I}}=1 \mathrm{~A}_{\mathrm{I}} \mathrm{V}$,
2 à $3 \mathrm{~A}_{\mathrm{I}} \mathrm{L}, 4$ à $5 \mathrm{~A}_{\mathrm{I}} \mathrm{D}$
$\mathrm{A}_{\text {II }}=$
3 à $6 \mathrm{~A}_{\mathrm{II}} \mathrm{L}, 4$ à $5 \mathrm{~A}_{\mathrm{II}} \mathrm{D}$
$\mathrm{A}_{\text {III }}=$
1 à $2 \mathrm{~A}_{\text {III }} \mathrm{L}, 3 \mathrm{~A}_{\mathrm{III}} \mathrm{D}$
$\mathrm{M}_{\mathrm{I}}=1 \mathrm{M}_{\mathrm{I}} \mathrm{V},=$
$3 \mathrm{M}_{\mathrm{I}} \mathrm{D}$
$\mathrm{M}_{\mathrm{II}}=2$ à $3 \mathrm{M}_{\mathrm{II}} \mathrm{V}$,
3 à $4 \mathrm{M}_{\mathrm{II}} \mathrm{D}$

- Acétabulum (fig. 3, D, E, F)

$\mathrm{S}=36$ à 39 papilles

- Quene (fig. 3, C)

Elle porte au total 58 à 63 papilles soit :

$\mathrm{U}=4 \mathrm{UD}, 50$ à $54 \mathrm{UDL}, 4$ parfois $5 \mathrm{U}$ terminales.

Le nitrate imprègne aussi les pores excréteurs caudaux.

\section{III - Comparaison avec les structures correspondantes déjà écrites}

\section{1 - Les miracidiums}

Les structures argyrophiles du miracidium de $P$. leydeni sont identiques à celles des miracidiums précédemment décrits de Liorchis scotiae, $P$. togolense, $P$. phillerouxi, P. daubneyi et P. microbothrium, cf. Katkov (1970), Albaret et coll. (1978), Albaret et coll. (1981), Samnaliev et coll. (1982), Diaw et col.. (1983) ; elles sont différentes de celles observées sur le miracidium de Calicophoron calicophorum par Swart (1967). Cette constatation ne permet pas de retenir la proposition d'Eduardo (1982b) qui consiste à classer les espèces togolense, phillerouxi, microbothrium et daubneyi dans le genre Calicophoron et les espèces leydeni et cervi dans le genre Paramphistomum.

\section{2 - Les cercaires}

Latkov (1973) publie la description des sensilles d'une cercaire déterminée Liorchis scotiae. Les renseignements fournis ne sont guère utilisables car l'auteur n'a pas fait de dessins à la chambre claire. Le schéma de la vue apicale qu'il donne, avec des papilles réparties selon huit axes subsymétriques ne correspond pas à ce que l'on observe chez les Paramphistomidae; et il semble, en outre, pour la figure du corps et de la queue, avoir confondu face ventrale et face dorsale. Toutefois le nombre total de papilles céphaliques et caudales qu'il indique correspond assez bien au nombre compté sur notre matériel. 
Outre cette description, nous possédons les chétotaxies de :

- Paramphistomum togolense parasite de Bulinus forskalii (hôte naturel et expérimental) et Ovis aries (hôte expérimental), cf. Albaret et coll. (1978)

- Paramphistomum phillerouxi parasite de Bulinus forskali (hôte expérimental) et Bos taurus (hôte naturel), cf. Albaret et coll. (1981)

- Paramphistomum daubneyi parasite de Lymnaea truncatula (hôte naturel et expérimental) et Bos taurus (hôte expérimental), cf. Samnaliev et coll. (1982)

- Paramphistomum microbothrium dont nous possédons deux souches : l'une parasite d'Isidora guernei (hôte naturel et expérimental) et Ovis aries (hôte expérimental), l'autre parasite d'Isidora truncata (hôte expérimental) et Bos taurus (hôte naturel), cf. Diaw et coll. (1983).

La comparaison de ces chétotaxies met en évidence quelques niveaux identiques ou proches : les niveaux $\mathrm{C}_{\mathrm{II}}$ et $\mathrm{AM} \mathrm{V}$ et quelques niveaux différents : les niveaux $\mathrm{S}$ et $\mathrm{U}$.

Chétotaxie acétabulaire : niveau $S$

La chétotaxie acétabulaire permet de diviser les cinq espèces considérées en trois groupes :

a) le groupe $P$. leydeni qui possède 36 à 39 papilles $\mathrm{S}$.

b) le groupe $P$. microbithrium - $P$. daubneyi qui possède 18 à 22 papilles ( $P$. microbothrium) ou 18 à 27 papilles ( $P$. daubneyi).

c) le groupe $P$. togolense $-P$. phillerouxi qui possède 5 à 10 papilles ( $P$. phillerouxi) ou 12 papilles (P. togolense).

Chétotaxie caudale : niveau $U$

La chétotaxie caudale permet la séparation en trois groupes qui ne se superposent pas aux précédents :

a) le groupe $P$. microbothrium - P. leydeni qui possède 51 à 65 papilles U.

b) le groupe $P$. togolense - $P$. phillerouxi qui possède 37 à 47 papilles.

c) le groupe $P$. daubneyi qui possède 9 à 19 papilles.

\section{Conclusion}

Deux séries d'informations peuvent être tirées de cette étude :

1 - Les miracidiums de Paramphistomum leydeni, P. togolense, P. phillerouxi, $P$. daubneyi et $P$. microbothrium se ressemblent entre eux et sont différents du miracidium de Calicophoron calicophorum.

Les cinq cercaires correspondantes ont plusieurs niveaux identiques ou proches en $\mathrm{C}_{\mathrm{II}}$ et $\mathrm{AM} \mathrm{V}$. 
Les analogies que ces cinq espèces présentent tant au niveau miracidial qu'au niveau cercarien nous paraissent apporter des arguments en faveur du bien fondé du genre Paramphistomum tel qu'il est admis par Näsmark (1937) et ne nous permettent pas de retenir une proposition d'Eduardo 1982(b) : rattacher au genre Calicophoron quelques espèces précédemment rattachées au genre Paramphistomum, parmi elles : $P$. togolense, $P$. phillerouxi, $P$. daubneyi, $P$. microbothrium et conserver dans le genre Paramphistomum quelques espèces dont $P$. leydeni.

2 - Les caractéristiques chétotaxiques acétabulaires et caudales des cercaires telles qu'elles apparaissent dans ce travail permettent un diagnostic facile des cinq espèces considérées alors que la détermination des adultes au contraire soulève beaucoup de controverse.

Nous pouvons définir ainsi les cinq Paramphistomes :

- Paramphistomum togolense et P. phillerouxi sont des parasites de Bulinus, ont une chétotaxie caudale avec un nombre moyen de papilles (37 à $47 \mathrm{U}$ ) et une chétotaxie acétabulaire avec un petit nombre de papilles (5 à $12 \mathrm{~S}$ ).

Ces deux espèces relativement très proches, se reconnaissent à leurs papilles $\mathrm{M}$.

- Paramphistomum microbothrium est parasite d'Isidora, a une chétotaxie caudale avec un grand nombre de papilles (51 à $65 \mathrm{U}$ ) et une chétotaxie acétabulaire avec un nombre moyen de papilles (18 à $22 \mathrm{~S}$ ).

- Paramphistomum daubneyi est parasite de Lymnaea, a une chétotaxie caudale avec un petit nombre de papilles (9 à $19 \mathrm{U}$ ) et un nombre moyen de papilles acétabulaires (18 à $27 \mathrm{~S}$ ).

- Paramphistomum leydeni est parasite de Planorbis, a une chétotaxie caudale avec un grand nombre de papilles (58 à $63 \mathrm{U}$ ) et un grand nombre de papilles acétabulaires (36 à $39 \mathrm{~S})$.

\section{BIBLIOGRAPHIE}

Albaret J. L., Bayssade-Dufour Ch., Guilhon J., Kulo S. D., Picot H. : Cycle biologique de Paramphistomum togolense n. sp. (Trematoda, Paramphistomidae). Ann. Parasitol. Hum. Comp., 1978, s3, 495-510.

Albaret J. L., Bayssade-Dufour Ch., Diaw O. T., Vassillades G., Sey O., Gruner L. : Disposition des organites argyrophiles superficiels du miracidium et de la cercaire de Paramphistomum phillerouxi Dinnik, I96I (Trematoda, Paramphistomidae). Ann. Parasitol. Hum. Comp., I981, s6, 147-154.

Combes C., BAySSADE-Dufour Ch., CAssone J. : Sur l'imprégnation et le montage des cercaires pour l'étude chétotaxique. Ann. Parasitol. Hum. Comp., 1976, s1, 399-400.

Diaw O. T., Samnaliev P., Pino L. A., Bayssade-Dufour Ch., Albaret J. L., Vassiliades G. : Structures argyrophiles des formes larvaires de deux souches de Paramphistomum microbothrium : l'une parasite d'Isidora guernei et Ovis aries, l'autre parasite d'Isidora truncata et Bos taurus. Ann. Parasitol. Hum. Comp., I983, 58, 455-465.

Eduardo S. I. : The taxonomy of the family Paramphistomidae Fischoeder, I9or with special reference to the morphology of species occuring in ruminants. I. General considerations. Systematic Parasitology, 1982a, 4, 7-57.

Eduardo S. I. : The taxonomy of the family Paramphistomidae Fischoeder, I9oI with special reference to the morphology of species occuring in ruminants. II. Revision of the genus Paramphistomum Fischoeder, r9or. Systematic Parasitology, 1982b, 4, 189-238. 
Katkov M. V. : To the question of morphology of larvae of Liorchis scotiae (Willmott, I950) Velichko, 1966 (Trematoda, Paramphistomatidae). Trud. Vses. Inst. Gelmint., I970, I6, 97-102.

KatKov M. V. : An improvement of methods of discovering of sensillae on the body of Paramphistomatoidea cercariae (Trematoda : Paramphistomatoidea Stiles et Goldberger, 19ro). Trud. Vses. Inst. Gelmint., 1972, 20, 101-105.

Lynch J. E. : The miracidium of Heronimus chelydrae Mac Callum. Quart. J. microsc. Sci., London N.S., $1933,76,12-33$.

NASMark K. E. : A revision of the Trematode family Paramphistomidae. Zoologista Bidrag Fran Uppsala, 1937, $16,301-566$.

Samnaliev P., Bayssade-Dufour Ch., Albaret J. L., Dimitrov V., Kamburov P. : Structures argyrophiles tégumentaires du miracidium, de la rédie et de la cercaire de Paramphistomum daubneyi Dinnik, 1962 (Trematoda, Paramphistomidae). Ann. Parasitol. Hum. Comp., I981, 56, 155-166.

SWART P. J. : A study of epidermal plates of the miracidia of calicophoron calicophorum (Fishoeder, I90I) Näsmark, I937 and Paramphistomum microbothrium (Fishoeder, I9OI). Onderstepoort. $J$. Vet. Res., $1967,34,129-136$. 